\title{
The British Society of Urogynaecology
}

\author{
Peter L. Dwyer • Robert Freeman • Linda Cardozo
}

(C) The International Urogynecological Association 2011

The International Urogynecology Journal is proud to support and have the support of the British Society of Urogynaecology (BSUG) and its members. Strong national bodies are important to the development of urogynecology, and to sustaining international organisations such as IUGA and this journal. The BSUG has become a vibrant, energetic, all-inclusive society that could serve as a blueprint for the way any new national urogynecology society should develop and function. The BSUG has an excellent relationship with its mother society, the Royal College of Obstetricians and Gynaecologists, and provides good services to its members, the broader obstetrics and gynecology community and the British public.

We congratulate the BSUG on its achievements and are pleased to publish in this issue the abstracts of its annual scientific meeting in 2010.

We thank Linda Cardozo and Bob Freeman, two past chairmen of BSUG, for providing us with the following

\section{P. L. Dwyer $(\bowtie)$}

Department of Urogynecology, Mercy Hospital for Women, University of Melbourne,

Melbourne, Australia

e-mail: pdwyer@connexus.net.au

\section{R. Freeman}

Department of Urogynaecology, Plymouth Hospitals NHS Trust, Derriford Hospital,

Plymouth, Devon, UK

\section{R. Freeman}

Peninsula College of Medicine and Dentistry,

The John Bull Building,

16 Research Way, Derriford,

Plymouth, Devon, UK

\section{Cardozo}

Department of Urogynaecology, King's College Hospital,

London, UK account of the history and present activities of their society.

Peter L. Dwyer, Co-Editor-in-Chief, International Urogynecology Journal

\section{The BSUG}

History The British Society of Urogynaecology was formed in 2001 following a request from the then President of the Royal College of Obstetricians and Gynaecologists (RCOG), Bob Shaw. He wanted to establish specialist societies who could advise the RCOG in areas relating to their subspecialty. While he was keen to affiliate them to the College, this was initially rejected by the Council. Nonetheless, the College Officers realised the importance of a close working relationship with specialist societies and a College Officers/Specialist Societies Liaison Group was formed. This meets twice a year to discuss issues of common interest.

At the time of the request, apart from the Research Urogynaecology Society (RUGS) set up by Stuart Stanton some years previously, no British urogynaecological society existed. Linda Cardozo, with whom Bob Shaw had spoken, therefore proposed that one should be formed and approached Bob Freeman and Vik Khullar, Chairman and Secretary of RUGS at the time. They started working on a constitution and setting up a general committee/executive and subcommittees.

A name had to be chosen, and the one settled on was British Society of Urogynaecology (BSUG), as British Urogynaecology Association, or "BUGA" (sounds like a rude word in English!) for short, and British Urogynaecology Society, or "BUGS", were not thought appropriate!

The aims of BSUG were, and still are, to set and raise standards in urogynaecology and assist the RCOG in, producing clinical guidelines and standards, give advice on 
training (subspecialty training, advanced training skills modules/ATSM) and workforce planning, being a joint stakeholder for NICE (the National Institute for Clinical Excellence) and producing protocols for the Department of Health 18 week wait for first treatment. The BSUG wanted to help its members by means of education, audit and advice, and this has been achieved through the subcommittees' hard work on clinical guidelines, governance, training, audit, meetings and research. The Society also helps members by providing citations for those applying for higher awards by virtue of its nominating body status for the ACCEA (the UK clinical excellence awards scheme).

The Guidelines Subcommittee, currently chaired by Ayman Elnaqa (previously by Mark Slack), has helped produce guidelines for the RCOG and has also played a major part in the College's Guidelines and Audit Committee.

The Governance Subcommittee, now chaired by Liz Adams, has an important role in helping colleagues with governance and medico-legal issues while also helping with patient information sheets in combination with the RCOG. Its greatest achievement to date has been the accreditation of units for "service", proposed to the College by the previous subcommittee chairman, Simon Hill, in 2007. While the RCOG was unable to formally accredit jointly with BSUG, nonetheless the idea received a positive response, as accreditation promotes best practice, helps to implement national guidelines as well as the RCOG's Clinical Standards Proposals (written by BSUG), recognises the "team" (rather than the individual) and acknowledges non-subspecialty units which provide high-quality care. It might also be useful for NHS hospitals when competing for contracts with independent sector and private agencies. BSUG recommends that members consider applying for accreditation.

The Training Subcommittee has worked incredibly hard to set up a multi-disciplinary urodynamic training accreditation with urologists, physiotherapists, continence advisers, medical physicists and others. This is now a recognised and accepted system and credit must go to Phil Toozs-Hobson and his subcommittee for this achievement.

Another success has been the Surgical Audit Database, originally proposed by David Richmond when he was chairman of the Audit Subcommittee. The work undertaken by Paul Moran has been outstanding. The database gives members the opportunity to collect data for all prolapse and incontinence surgery. In addition it has the potential to become a national database which will be useful for all clinicians and patients. This database has been a high priority for BSUG, with significant funding provided to ensure it is available to all members free of charge.

Following on from RUGS, the Research Subcommittee, now chaired by Doug Tincello (previously by Abdul Sultan), organises an annual meeting where ongoing or new research can be presented for advice and feedback. In addition there has been collaboration with various groups, including the UK Comprehensive Research Network (UKCRN) national trials portfolio and pelvic floor clinical studies group, towards developing new protocols. Statistical and methodological advice is free to members.

Finally, the Meetings Subcommittee chaired by Tim Hillard (following Ash Monga and Ranee Thakar) has an annual meeting jointly with the RCOG in June or November in addition to other meetings, including a surgical course and one on obstetric pelvic floor injury. These are well attended and of high quality, often with international speakers.

Road shows Some years ago it was felt that the BSUG should visit obstetricians and gynaecologists throughout the UK to tell them about the Society, dispel some of the myths (especially about "elitism") and provide updates on best practice in urogynaecology. "Road shows" were arranged to explain the BSUG's role in setting and raising standards, through guidelines, promoting the RCOG's clinical standards, meetings, auditing outcomes through our database and, as an affiliate society to the IUGA, receiving the International Urogynecology Journal (IUJ). Most regions of the UK have been visited with good attendances and feedback.

IUGA The relationship with the IUGA has grown since Linda Cardozo was IUGA President in 1998-2000. In 2004 the BSUG formally affiliated to the IUGA and this has been beneficial to both societies: members receive the IUJ, which helps them to keep up to date, and IUGA membership has increased. Many BSUG members now attend the annual IUGA meeting and several are chairs of committees.

The future for BSUG is bright and the new committee is working hard on many areas which can be viewed on the excellent new website (http://www.bsug.org.uk)

The aims of the BSUG from the beginning have been to set and raise standards, and these goals, we feel, are being achieved. For example, the successful creation of new urogynaecology consultant posts has been most gratifying. In the past there were only one or two created in a year; since January 2010, however, there have been 23 new consultant posts with a special interest in urogynaecology in the UK. This will hopefully mean that more trainees will want to be trained in urogynaecology ultimately leading to widespread provision of high-quality evidence-based care for patients.

Through BSUG and its relationship with the RCOG, urogynaecology has become recognised as a major subspecialty in the UK.

Professor Robert Freeman: Founding Honorary Secretary and immediate Past Chairman

Professor Linda Cardozo: Founding Chairman. 